\title{
Effect of impregnation on hybrid mesoporous silica / kenaf reinforced epoxy composites in term of flexural, compressive and water absorption properties
}

\begin{abstract}
In this work, mesoporous silica with designated amount was added in kenaf/epoxy composites to improve the mechanical properties of the composite and reduce the water uptake of fabricated composites. The composites were fabricated using hot press method (HP) and impregnation method (IMP). For HP specimens, silica was dispersed into epoxy resin using homogenizer before being applied to the kenaf mat and subsequently hot pressed. While for IMP specimens, the kenaf mat was placed inside the silica/epoxy solution under $600 \mathrm{~mm} \mathrm{Hg}$ impregnation pressure before being hot pressed. The results for flexural properties revealed that IMP specimen with $40 \mathrm{vol} \%$ of kenaf fibre and 5 vol\% of silica have the highest strength and modulus at 78.6 MPa and 5.11 GPa respectively. Same trend can be seen for compressive properties as the same specimen had the highest compressive strength and modulus at $69.3 \mathrm{MPa}$ and $1.81 \mathrm{GPa}$ respectively. Finally for water absorption properties, IMP specimens had a reduction in water uptake compared to its HP specimen counterparts with the same kenaf and silica content. IMP specimen with $60 \mathrm{vol} \%$ kenaf and 5 vol\% silica had the lowest water uptake after 90 days of immersion in distilled water at $13.5 \%$ increase in weight.
\end{abstract}

Keyword: Kenaf; Mesoporous silica; Hybrid composites; Natural fibre reinforced composites 